\title{
Microstructure effect on firm's volatility risk
}

\author{
Flavia Barsotti * Simona Sanfelici \\ ISFA Department of Economics \\ University Lyon 1, France University of Parma, Italy \\ flavia.barsotti@univ-lyon1.fr simona.sanfelici@unipr.it \\ fl.barsotti@gmail.com
}

October 7, 2012

\begin{abstract}
Equity returns and firm's default probability are strictly interrelated financial measures capturing the credit risk profile of a firm. Following the idea proposed in [20] we use high-frequency equity prices in order to estimate the volatility risk component of a firm within Merton [17] structural model. Differently from [20] we consider a more general framework by introducing market microstructure noise as a direct effect of using noisy high-frequency data and propose the use of nonparametric estimation techniques in order to estimate equity volatility. We conduct a simulation analysis to compare the performance of different non-parametric volatility estimators in their capability of i) filtering out the market microstructure noise, ii) extracting the (unobservable) true underlying asset volatility level, iii) predicting default probabilities calibrated from Merton [17] model.
\end{abstract}

Keywords: market microstructure noise, high-frequency data, non-parametric volatility estimation, Merton model, default probabilities, volatility risk.

\section{Introduction}

In structural credit risk models, firstly formalized by [17], a firm's equity and debt can be seen as contingent claims partitioning the asset value of the firm. Empirical tests of structural credit risk models show poor predictions of default probabilities and credit spreads, especially when considering short maturities. Methods of strict

*Part of this research has been financed with a grant by University of Florence during my Post-Doc position at the Department of Mathematics for Decisions. 
estimation or calibration provide evidence that predicted credit spreads are far below observed ones [14], the structural variables explain little of the credit spread variation [12], and pricing error is large for corporate bonds [18]. A critical issue about the implementation of these models is that firm's asset value and volatility are not directly observable. The idea is then to use the information content of equity prices and then back out the firm's asset volatility. Nevertheless, the market microstructure literature strongly suggests that trading noises can affect equity prices so that the estimation of equity volatility and other related quantities may become a difficult task.

As well documented, observed equity prices can diverge from their equilibrium value due to illiquidity, asymmetric information, price discreteness and other measurement errors. For example, the effects of trading noise on how frequently one should sample the equity price are analyzed in $[4,2]$.

In the specific context of structural credit risk models, the relationship between the unobservable asset volatility and the observed equity value predicted by the pricing model is masked by trading noise; ignoring microstructure effects could nontrivially inflate estimates for the "true" asset volatility, and this would produce misleading estimates for default probabilities and credit spreads. This issue has been analyzed in [9], where the authors extend [8] to explicitly account for trading noise contamination of equity prices: they devise a particle filter-based maximum likelihood method based solely on the time series of observed equity values, which is robust to market microstructure effects. The importance of using high frequency data to back out parameters involved in firm's value dynamics has been highlighted by [20], which propose a novel approach to identify the volatility and jump risks component of individual firms from high-frequency equity prices. Their analysis suggests that high-frequency-based volatility measures can help to better explain credit spreads, above and beyond what is already captured by the true leverage ratio. However, the highest frequency considered in this paper is the 5 -minute conservative sampling frequency which allows to eliminate microstructure effects.

In this paper we propose a particular econometric approach to structural models calibration based on a non-parametric estimation of equity volatility from highfrequency intra-day equity prices. Several non-parametric estimators of daily stock volatility have been proposed in the econometric literature allowing to exploit the information contained in intra-day high frequency data neglecting microstructure effects $[1,4,19,11,2,13,15]$. We propose a Monte Carlo simulation study based on Merton [17] structural model trying to compare the performance of different non-parametric volatility estimators in their capability of i) filtering out the market microstructure noise, ii) extracting the true underlying asset (unobservable) volatility level, iii) predicting default probabilities. Our analysis shows that the choice of the volatility estimator can largely affect calibrated default probabilities and hence risk evaluation. In particular, the commonly used Realized Volatility estimator is unable to provide reliable estimates for the volatility risk leading to a significant underestimation of default probabilities. 


\section{Merton model under microstructure effects}

In this Section we describe the main features of Merton [17] credit risk structural model and provide the theoretical definition of each economic quantity and volatility estimator useful for our simulation analysis. In Subsection 2.1 we characterize firm's asset value evolution and in Subsection 2.2 we describe equity volatility estimation procedure under market microstructure noise.

\subsection{Firm value model}

We follow Merton [17] structural model by assuming a firm's value process described by a geometric Brownian motion which evolves according to

$$
\frac{d A_{t}}{A_{t}}=(\mu-\delta) d t+\sigma d W_{t}
$$

where $A_{t}$ is asset value at time $t, \mu$ is the instantaneous asset return, $\delta$ the asset payout ratio and $\sigma$ the asset volatility. The firm has two classes of claims outstanding: equity and a zero-coupon debt with maturity $T$. To be suitable for pricing corporate debt, we adopt the following bankruptcy assumptions from [17]: (i) the firm issues one zero-coupon bond with a promised payment $B$ at maturity, (ii) default occurs only at maturity with debt face value as default boundary, and (iii) when default occurs, the absolute priority rule prevails. The payoffs to debt holders and equity holders at time $T$ become, respectively:

$$
D_{T}=\min \left(A_{T}, B\right), \quad S_{T}=\max \left(A_{T}-B, 0\right) .
$$

From now on, we focus our attention on equity value and default probabilities in order to develop our computational econometric analysis. Equity claim can be priced at each time $t<T$ through the standard Black-Scholes option pricing model as the price of a European call option given by

$$
S_{t}=S\left(A_{t} ; \sigma, B, r, \delta, T\right)=A_{t} \phi\left(d_{t}\right)-B e^{-r(T-t)} \phi\left(d_{t}-\sigma \sqrt{T-t}\right),
$$

where

$$
d_{t}:=\frac{\log \left(\frac{A_{t}}{B}\right)+\left(r+\sigma^{2} / 2\right)(T-t)}{\sigma \sqrt{T-t}},
$$

and $\phi(\cdot)$ is the standard normal distribution function. Therefore, by applying Itô's lemma, the instantaneous volatility $\Sigma_{t}^{s}$ of the log equity price can be written as:

$$
\Sigma_{t}^{s}:=\frac{A_{t}}{S_{t}} \frac{\partial S_{t}}{\partial A_{t}} \sigma
$$

Notice that the equity volatility is driven by the time-varying factor $A_{t}$, whereas the asset volatility $\sigma$ is constant. The firm's probability of default at maturity $T$ is the probability of $A_{T}$ being below the constant barrier represented by the face value of debt $B$. Under the physical probability measure $\mathbb{P}$ we have

$$
\mathbb{P}\left(A_{T}<B \mid A_{t}\right)=\phi\left(\sigma \sqrt{T-t}-d_{t}^{\mathbb{P}}\right),
$$

with $d_{t}^{\mathbb{P}}$ given by Equation (3) where we only replace the interest rate $r$ with $\mu-\delta$. 


\subsection{Equity volatility estimation}

For a given firm, one can obtain a time series of equity prices $\left\{S_{j}, j=0, \ldots, N\right\}$, with a given sampling frequency $h=t_{j}-t_{j-1}$ assumed to be constant, for ease of exposition. If one could observe the "true" equity price, than equity volatility could be easily estimated by the well known Realized Volatility estimator [7] at any desired accuracy level using high frequency data. However, as noted for instance by [9], the time series of observed equity prices may contain trading noises, hence volatility estimation becomes much more complex. In particular, the relationship between the unobserved asset and the observed equity value predicted by the pricing formula (2) may be masked by trading noise. We assume an additive error structure for the trading noise on the logarithmic equity value as follows

$$
\log \widetilde{S}\left(t_{j}\right)=\log S\left(t_{j}\right)+\eta\left(t_{j}\right)
$$

where the random shocks $\eta\left(t_{j}\right)$, for $0 \leq j \leq N$ are i.i.d. random variables with mean zero and bounded fourth moment and independent of the efficient log-return process. The assumption of independence can be relaxed by considering a particular form of dependent noise, given by [11], with market microstructure noise that is time-dependent in tick time and correlated with efficient returns

$$
\widetilde{\eta}_{j}:=\alpha\left[\log S\left(t_{j}\right)-\log S\left(t_{j-1}\right)\right]+\eta_{j},
$$

where $\alpha$ is a real constant and $\widetilde{\eta}_{j}$ and $\eta_{j}$ are the shorten notation for $\widetilde{\eta}\left(t_{j}\right)$ and $\eta\left(t_{j}\right)$. The case $\alpha=0$ corresponds to the case of independent noise assumption.

The basic idea of our paper is that, using suitable volatility estimators, we can infer the true volatility process $\Sigma_{t}^{s}$ of equity returns from noisy high-frequency data. Then, the equity volatility estimate can be used to back out the asset volatility $\sigma$ such as to fit exactly, say, the 5-years probability of default by solving Equation (5) with respect to $\sigma$, as explained in details in the following section.

For the reader's convenience, we now give some details about the implementation of the volatility measures employed in our analysis. We set $\tilde{p}_{t}:=\log \widetilde{S}_{t}$, the noisy equity log-price. Time is measured in daily units. We build daily measure of volatility by considering daily windows of $n$ intra-day equity data $\tilde{p}_{t, j}, j=0,1, \ldots, n$. Besides the well known Realized Volatility estimator

$$
\Sigma_{t}^{R V}:=\sum_{j=1}^{n} \delta_{j}(\tilde{p})^{2}
$$

where $\delta_{j}(\tilde{p}):=\tilde{p}_{t, j}-\tilde{p}_{t, j-1}$ is the $j$-th within-day equity log-return on day $t$, we consider the following estimators of the volatility process $\Sigma_{t}^{s}$ : the bias corrected estimator by Hansen and Lunde [11]

$$
\Sigma_{t}^{H L}:=\Sigma_{t}^{R V}+2 \frac{n}{n-1} \sum_{j=1}^{n-1} \delta_{j}(\tilde{p}) \delta_{j+1}(\tilde{p})
$$

the flat-top realized kernels by [4], [6]

$$
\Sigma_{t}^{K}:=\sum_{h=-H}^{H} k\left(\frac{h}{H+1}\right) \sum_{j=|h|+1}^{n} \delta_{j}(\tilde{p}) \delta_{j-|h|}(\tilde{p}),
$$


with kernels of $\mathrm{TH}_{2}$ type $k(x)=\sin ^{2}\left(\frac{\pi}{2}(1-x)^{2}\right)$. The realized kernels may be considered as unbiased corrections of the Realized Volatility by means of the first $H$ autocovariances of the returns. In particular, when $H$ is selected to be zero the realized kernels become the Realized Volatility. Our analysis includes also the twoscale estimator by [19]

$$
\Sigma_{t}^{T S}:=\frac{S}{S-1}\left(\frac{1}{S} \sum_{s=1}^{S} \Sigma_{t}^{G^{(s)}}-\frac{1}{S} \Sigma_{t}^{R V}\right)
$$

The two-scale (subsampling) estimator is a bias-adjusted average of lower frequency realized volatilities computed on $S$ non-overlapping observation subgrids $G^{(s)}$ containing $n_{S}$ observations. Recently, [13] proposed a pre-averaging technique as an alternative to subsampling in order to reduce the microstructure effects. The idea is that if one averages a number of observed log-prices, one is closer to the latent process $p(t)$. This approach, when well implemented, gives rise to rate optimal estimators of power variations. In particular, a consistent estimator of the integrated volatility can be constructed as

$$
\Sigma_{t}^{P A}=\frac{\sqrt{\Delta}}{\theta \psi_{2}} \sum_{s=0}^{n-k_{n}+1} \bar{\delta}_{s}(\tilde{p})^{2}-\frac{\psi_{1} \Delta}{2 \theta^{2} \psi_{2}} \sum_{s=1}^{n} \delta_{s}(\tilde{p})^{2}
$$

where the pre-averaged return process is given by

$$
\bar{\delta}_{s}(\tilde{p}):=\sum_{r=1}^{k_{n}} g\left(\frac{r}{k_{n}}\right) \delta_{s+r}(\tilde{p})=\frac{1}{k_{n}}\left(\sum_{j=k_{n} / 2}^{k_{n}-1} \tilde{p}_{t, s+j}-\sum_{j=0}^{k_{n} / 2-1} \tilde{p}_{t, s+j}\right),
$$

$\theta=k_{n} \sqrt{\Delta}, \psi_{1}=1$ and $\psi_{2}=1 / 12$, corresponding to the "hat" weight function $g(x)=x \wedge(1-x)$. The Fourier estimator [15] is given by

$$
\Sigma_{t}^{F}=\frac{(2 \pi)^{2}}{2 N+1} \sum_{s=-N}^{N} c_{s}\left(d \tilde{p}_{n}\right) c_{-s}\left(d \tilde{p}_{n}\right),
$$

where $c_{k}\left(d \tilde{p}_{n}\right)=\frac{1}{2 \pi} \sum_{i=1}^{n} \exp \left(-\mathrm{i} k t_{i-1}\right) \delta_{i}(\tilde{p})$. Finite sample MSE-based optimal rules for choosing the parameters employed by these estimators are discussed in [3], [19], [16] and [5]. Here, we proceed according to the following rules: a simple approximation of the optimal sampling frequency for the Realized Volatility estimator is to choose the number of observations approximately equal to $n^{*}=\left(Q / 4 E\left[\eta^{2}\right]^{2}\right)^{1 / 3}$, where $Q$ is the integrated quarticity estimated by means of low frequency returns. The optimal number of sub-grids $S$ is given by $c^{*} n^{2 / 3}$, where $c^{*}=\left(Q / 48 E\left[\eta^{2}\right]^{2}\right)^{-1 / 3}$. For the Kernel estimator, we apply the optimal mean square error bandwidth selection suggested by [5] and get $H=c^{*} \xi^{4 / 5} n^{3 / 5}$, where $c^{*}=(144 / 0.269)^{1 / 5}$, $\xi^{2}=E\left[\eta^{2}\right] / \sqrt{Q}$. In the case of the Pre-averaging estimator, inspired by [5], we choose $k_{n}=c^{*} \xi^{4 / 5} n^{3 / 5}$. Finally, for the Fourier estimator, the optimal cutting frequency $N$ can be easily obtained by direct minimization of the estimated MSE given by Theorem 3 in [16]. 


\section{Monte Carlo simulation}

In this section we provide numerical results of our Monte Carlo simulation analysis.

The aim is to compare the performance of different volatility estimators when using high-frequency equity data and equity prices are affected by trading noise. We analyze the performance of the volatility estimators with respect to their ability of i) filtering the microstructure noise and correctly extract equity volatility, ii) backing out asset volatility and iii) predicting default probabilities.

We perform a Monte Carlo simulation analysis in which the underlying asset dynamic is generated according to model (1). Then, high-frequency equity prices are simulated through Equation (2). The sample contains 500 days and equi-spaced intra-day data are generated at a frequency $h=4$ sec (i.e. $n=21600)$. The model parameters are $\sigma=0.2128, \mu=0.0643, B=43.13, r=0.05$ and $\delta=0.02$, as calibrated by [20] for bonds belonging to rating class A. Then, market microstructure noise is considered, alternatively, for both cases described by equations (6)-(7). The random shocks $\eta_{j}$ are i.i.d Gaussian random variables with mean zero and standard deviation equal to 2 times the standard deviation of log-equity returns. In the dependent noise case (7), we set $\alpha=0.5$. Once we have noisy equity prices, we compare the performance of different volatility estimators in their ability of extracting the "true" equity volatility $\Sigma_{t}^{s}$ given in (4) and other related quantities for the underlying process describing firm's assets value dynamics.

Table 1 presents numerical evidence for each equity volatility estimator introduced in subsection 2.2 and used in our comparison when (a) trading noise is independent of intra-day equity log-returns and (b) trading noise is correlated with intra-day equity log-returns, respectively.

The table lists the average relative error (ARE), the mean squared error (MSE) and bias achieved by the different equity volatility estimators. $\Sigma_{t}^{R V}$ represents the Realized Volatility estimator using all tick-by-tick equity data as given by Equation (8), while $\Sigma_{t}^{R V S S}$ refers to the Realized Volatility estimator based on sparse sampling, where the sampling frequency is optimized in order to filter the microstructure effects, by setting $n=n^{*}$ as explained in subsection 2.2. Our results strongly confirm well known stylized facts documented by the econometric literature and highlighted by [16]: $\Sigma_{t}^{R V}$ estimates are completely swamped by noise and sparse sampling can only moderately provide efficient estimates. The first order correction of $\Sigma_{t}^{H L}$ estimator given in Equation (9), as an alternative to sparse sampling, can reduce the bias due to the spurious first order autocorrelation in equity returns introduced by the trading noise. The best results are provided by $\Sigma_{t}^{F}$ and by the other estimators specifically designed to handle microstructure effects $\Sigma_{t}^{T S}, \Sigma_{t}^{K}$ and $\Sigma_{t}^{P A}$. However, the rank of estimators is different when we consider absolute error measures such as MSE and bias versus percentage error measures such as ARE. In fact, in the latter case $\Sigma_{t}^{H L}$ performs better than $\Sigma_{t}^{P A}$ and $\Sigma_{t}^{F}$. Finally, we notice that $\Sigma_{t}^{P A}$ tends to underestimate volatility, differently from all the other estimators.

In order to study the influence of different equity volatility estimators on the default probability predicted by Merton [17] model, we proceed by developing the following calibration exercise. For each equity volatility estimator, generically de- 
Table 1: Equity Volatility Estimators. The table shows average relative error (ARE), mean squared error (MSE) and BIAS for different equity volatility estimators. Results are based on 500 daily Monte Carlo simulations. Panel (a) refers to the trading noise given in Equation (6), panel (b) to Equation (7), with $\alpha=0.5$.

\begin{tabular}{lrrr}
\hline (a) & ARE & MSE & BIAS \\
\hline$\sum_{t}^{R V}$ & $2.00 \mathrm{e}+0$ & $4.49 \mathrm{e}-1$ & $6.69 \mathrm{e}-1$ \\
$\sum_{t}^{R V S S}$ & $3.28 \mathrm{e}-2$ & $4.13 \mathrm{e}-4$ & $1.10 \mathrm{e}-2$ \\
$\Sigma_{t}^{T S}$ & $5.37 \mathrm{e}-4$ & $2.55 \mathrm{e}-5$ & $1.97 \mathrm{e}-4$ \\
$\Sigma_{t}^{H L}$ & $9.89 \mathrm{e}-4$ & $2.21 \mathrm{e}-4$ & $3.73 \mathrm{e}-4$ \\
$\sum_{t}^{K}$ & $5.10 \mathrm{e}-4$ & $5.62 \mathrm{e}-5$ & $1.83 \mathrm{e}-4$ \\
$\Sigma_{t}^{P A}$ & $-1.19 \mathrm{e}-3$ & $6.59 \mathrm{e}-5$ & $-3.87 \mathrm{e}-4$ \\
$\Sigma_{t}^{F}$ & $1.33 \mathrm{e}-2$ & $8.01 \mathrm{e}-5$ & $4.46 \mathrm{e}-3$ \\
\hline$(\mathrm{b})$ & ARE & MSE & BIAS \\
\hline$\Sigma_{t}^{R V}$ & $2.24 \mathrm{e}+0$ & $5.65 \mathrm{e}-1$ & $7.50 \mathrm{e}-1$ \\
$\Sigma_{t}^{R V S S}$ & $3.61 \mathrm{e}-2$ & $4.90 \mathrm{e}-4$ & $1.21 \mathrm{e}-2$ \\
$\Sigma_{t}^{T S}$ & $6.31 \mathrm{e}-4$ & $2.79 \mathrm{e}-5$ & $1.96 \mathrm{e}-4$ \\
$\Sigma_{t}^{H L}$ & $1.07 \mathrm{e}-3$ & $3.25 \mathrm{e}-4$ & $3.46 \mathrm{e}-4$ \\
$\Sigma_{t}^{K}$ & $6.29 \mathrm{e}-4$ & $5.78 \mathrm{e}-5$ & $2.03 \mathrm{e}-4$ \\
$\sum_{t}^{P A}$ & $-1.43 \mathrm{e}-3$ & $6.79 \mathrm{e}-5$ & $-4.81 \mathrm{e}-4$ \\
$\Sigma_{t}^{F}$ & $1.38 \mathrm{e}-2$ & $8.58 \mathrm{e}-5$ & $4.60 \mathrm{e}-3$ \\
\hline
\end{tabular}


noted by $E$, and each day $t$ in our sample, we find the corresponding asset volatility estimate $\hat{\sigma}^{E}$ by matching the 5 -years default probability coming from our equity volatility estimate $\Sigma_{t}^{E}$ with the one evaluated through the model using the given parameters values. In so doing, we act as if we did not know the asset values to mimic the real-life estimation situation and we conduct inference only based on observable quantities such as measurable equity volatility and the 5 -years default probabilities. Default probabilities are computed, for any maturity, according to Equation (5). In order to avoid arbitrage opportunities, following [20], we consider as key assumption that all securities written on the underlying firm value $A_{t}$ must have the same Sharpe ratio, see [17], Equation (6). This consideration enables us to express the instantaneous asset return $\mu$ as a function of the unknown asset volatility, given each equity volatility estimate $\Sigma_{t}^{E}$, and then to solve Equation (5) for the 5-years default probability with respect to the asset volatility, to obtain the corresponding asset volatility estimate $\hat{\sigma}^{E}$. Once asset volatility is known, we can compute default probabilities for any other maturity according to Equation (5).

Before analyzing the impact of the different volatility estimators on the calibration procedure for default probabilities, we analyze their influence on the calibration of asset volatility $\sigma$. A visual insight on the variability of asset volatility estimates obtained with the procedures based on the different equity volatility estimators can be achieved from Figure 1, plotting the ratio $\hat{\sigma}^{E} / \sigma$ in the case of independent noise. It is evident how the high-frequency Realized Volatility procedure (black line in the bottom) largely underestimate asset volatility. Similar results are obtained for the dependent noise case, as Figure 2 shows.

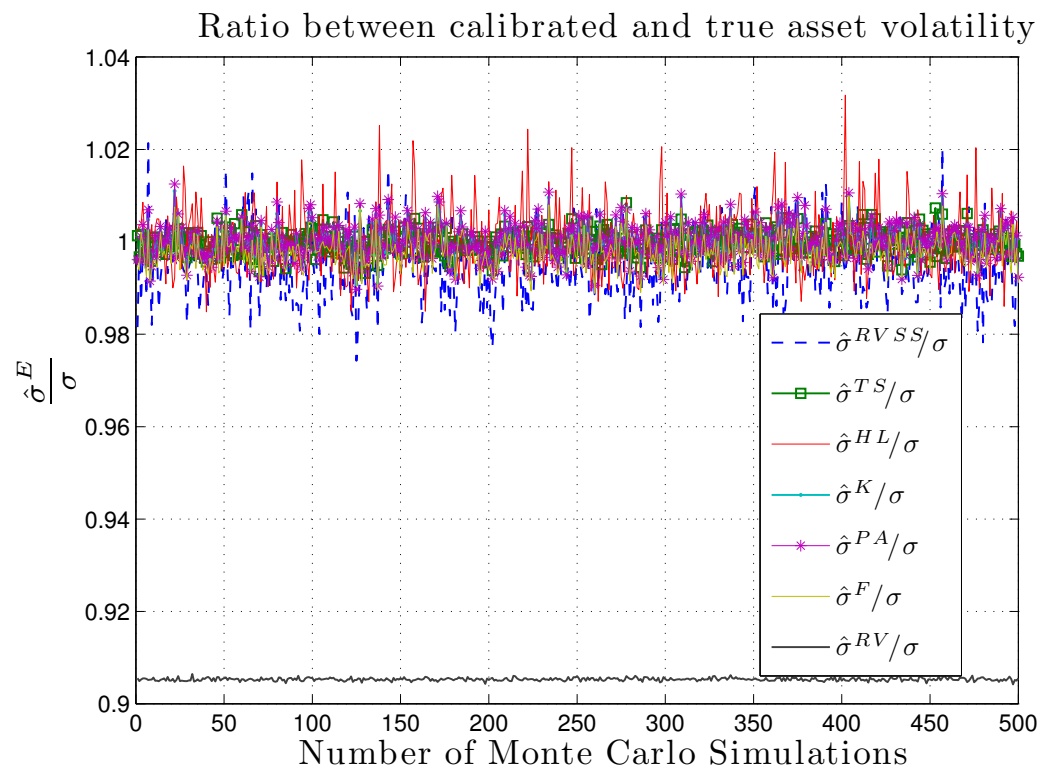

Figure 1: Calibrated Asset Volatility. The plot shows the ratio between calibrated asset volatility $\hat{\sigma}^{E}$ and the true asset volatility $\sigma$ for 500 daily Monte Carlo simulations. The plot refers to the trading noise given in Equation (6). Panel (a) of Table 2 gives descriptive statistics of these values. 
Table 2 shows descriptive statistics of calibrated asset volatility. We report results obtained by matching 5 -years default probabilities for each equity volatility estimator $E$. Panel (a) of the table refers to a trading noise of the form (6); panel (b) refers to results obtained for a trading noise of the form (7). It is evident from the table that the Kernel an Two-scale estimators provide the most accurate estimation of the true value $\sigma=0.2128$, with the smallest standard deviation. On the contrary, $\hat{\sigma}^{R V}$ is strongly biased due to microstructure effects, while the optimized $\hat{\sigma}^{R V S S}$ is less biased, at the price of a slightly larger variance. These results are confirmed by statistics for the ratio $\hat{\sigma}^{E} / \sigma$ as well.

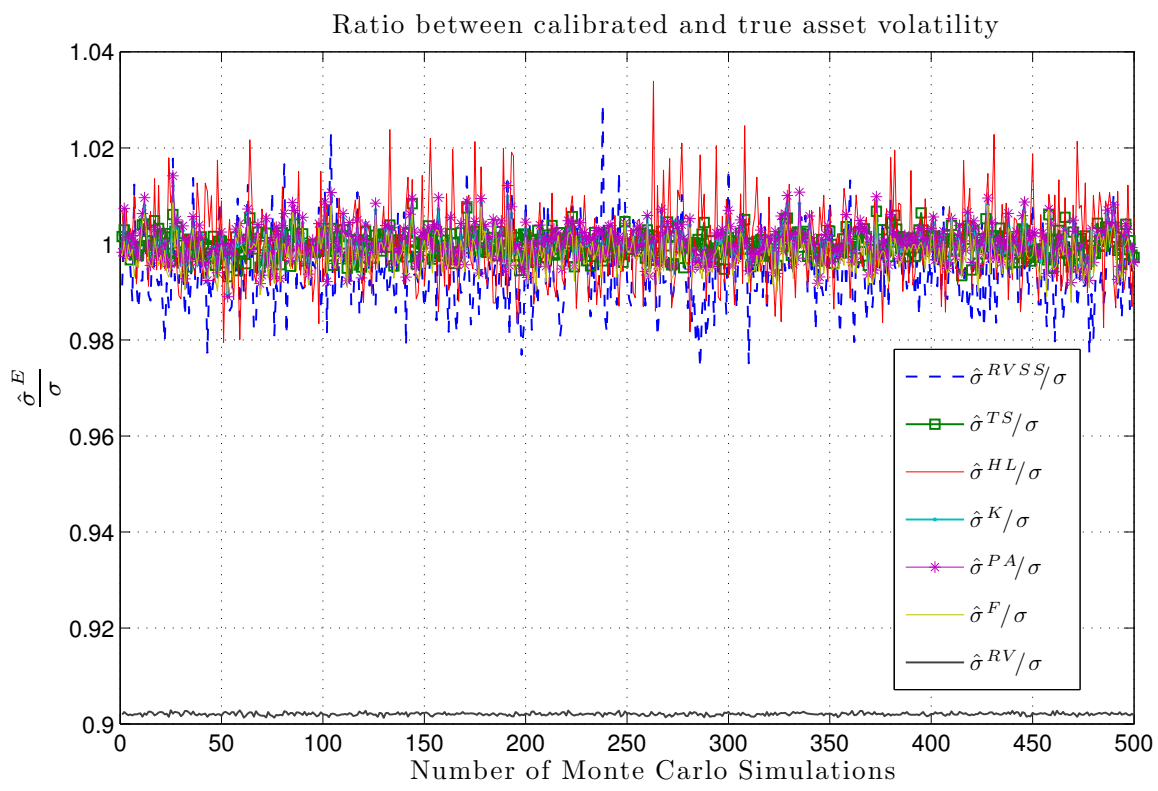

Figure 2: Calibrated Asset Volatility. The plot shows the ratio between calibrated asset volatility $\hat{\sigma}^{E}$ and the true asset volatility $\sigma$ for 500 daily Monte Carlo simulations. The plot refers to the trading noise given in Equation (7), with $\alpha=0.5$. Panel (b) of Table 2 gives descriptive statistics of these values.

The average (over all the 'daily' Monte Carlo replications) default probabilities for different maturities and calibration procedures are plotted in Figure 3.

For each day in the sample and for each volatility estimator $E$ we use the calibrated asset volatility $\hat{\sigma}^{E}$ in order to compute the default probabilities for any maturity (from 1 to 5 years) by Equation (5). From the figure, it appears evident how the Realized Volatility approach based on high frequency noisy data drastically underestimates default probabilities, so that sparse sampling becomes mandatory when equity data are affected by microstructure effects. On the whole, all the other procedures seem to provide sensible results and only a deeper analysis reveals differences among different calibration procedures.

The analysis of the effects of using alternative equity volatility estimators on default probabilities is conducted for different maturities (from 1 to 5 years) through 
Table 2: Calibrated Asset Volatility. The table shows descriptive statistics of calibrated asset volatility for different equity volatility estimators. Results are based on 500 daily Monte Carlo simulations. Panel (a) refers to the trading noise given in Equation (6), panel (b) to Equation (7), with $\alpha=0.5$.

\begin{tabular}{|c|c|c|c|c|c|c|c|}
\hline (a) & $\hat{\sigma}^{R V}$ & $\hat{\sigma}^{R V}$ & $\hat{\sigma}^{T S}$ & $\hat{\sigma}^{H L}$ & $\hat{\sigma}^{K}$ & $\hat{\sigma}^{P A}$ & $\hat{\sigma}^{F}$ \\
\hline Mean & 92652 & .211811 & 12759 & 212812 & 212770 & .212830 & 212355 \\
\hline & 2648 & & 2750 & 18 & 2734 & 212790 & 12313 \\
\hline & 2569 & & 212129 & 211089 & 211934 & 211896 & 211390 \\
\hline & 92743 & 0 & 213361 & 214912 & 3688 & 213878 & 213298 \\
\hline Min & 92419 & 1 & 11354 & 209575 & & 210616 & 10435 \\
\hline Max & 92894 & & 4601 & .219557 & & 15468 & 14927 \\
\hline \multirow[t]{2}{*}{ Std Deviation } & 00069 & & 0503 & 01515 & 0742 & 000807 & 000749 \\
\hline & $\hat{\sigma}^{R V} / \sigma$ & & $\hat{\sigma}^{T S} / \sigma$ & $\hat{\sigma}^{H L} / \sigma$ & $\hat{\sigma}^{K} / \sigma$ & $\hat{\sigma}^{P A} / \sigma$ & $\hat{\sigma}^{F} / \sigma$ \\
\hline 1010 & 905318 & & .999808 & 00056 & 999860 & 00139 & 997911 \\
\hline & & & & & & & 7711 \\
\hline & & & & & & & 3376 \\
\hline & & & & & & & 341 \\
\hline X & & & & & & & 885 \\
\hline & & & & & & & 996 \\
\hline Std & 26 & & & & 488 & 792 & 3519 \\
\hline (b) & $\hat{\sigma}^{R V}$ & & $\hat{\sigma}^{T S}$ & $\hat{\sigma}^{H L}$ & $\hat{\sigma}^{K}$ & $\hat{\sigma}^{P A}$ & $\hat{\sigma}^{F}$ \\
\hline Mea & 91966 & .211722 & 2756 & 12841 & 2766 & 12838 & 2340 \\
\hline & & & & & & & 320 \\
\hline & & & & & & & 1347 \\
\hline & & & & & & & 3360 \\
\hline & & & & & & & 0210 \\
\hline & & & & & & & 0.214626 \\
\hline \multirow[t]{2}{*}{ Std D } & 0.000062 & & .000524 & 0.001801 & 000755 & .000823 & 0.000774 \\
\hline & $\hat{\sigma}^{R V} / \sigma$ & $\hat{\sigma}^{R V}$ & $\hat{\sigma}^{T S} / \sigma$ & $\hat{\sigma}^{H L} / \sigma$ & $\hat{\sigma}^{K} / \sigma$ & $\hat{\sigma}^{P A} / \sigma$ & $\hat{\sigma}^{F} / \sigma$ \\
\hline & 97 & & & & 99840 & 0177 & 997840 \\
\hline & & & & & & & 7745 \\
\hline & & & & & & 82 & 3174 \\
\hline & & & & & & & 2631 \\
\hline & & & & & 9710 & 9029 & 0.987827 \\
\hline & & & & & & & 1.008582 \\
\hline Std Deviation & .000293 & 0.00 & 0.002462 & 0.008463 & 0.003550 & 0.003869 & 0.003638 \\
\hline
\end{tabular}




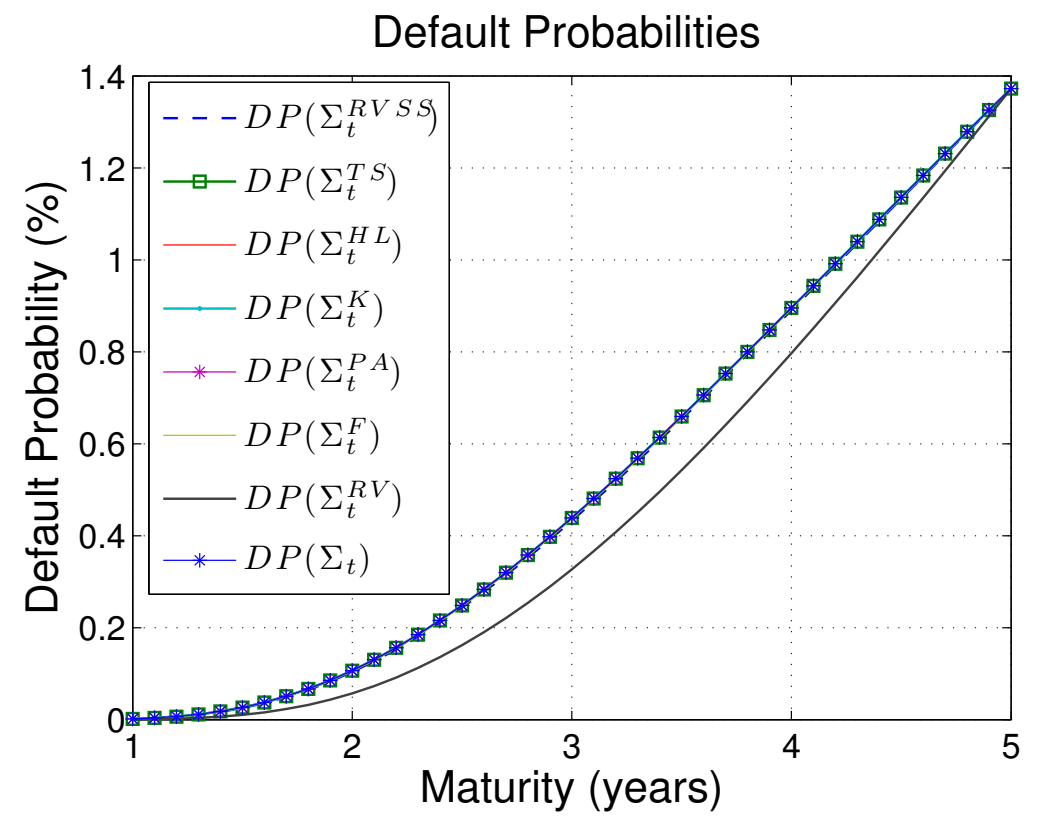

Figure 3: Default Probability. The plot shows default probabilities given by (5). By matching 5-years default probability coming from our equity volatility estimate with the one evaluated through the model using true parameters values, we obtain the corresponding calibrated asset asset volatility.

the comparison of the mean relative error between the estimated default probability and the theoretical one. For each maturity, we consider the following measure

$$
D P_{E r r}^{E}:=\mathbb{E}\left[\frac{D P\left(\Sigma_{t}^{E}\right)-D P\left(\Sigma_{t}^{s}\right)}{D P\left(\Sigma_{t}^{s}\right)}\right] \cdot 100,
$$

where $\operatorname{DP}\left(\Sigma_{t}^{E}\right)$ is the default probability calibrated from Merton [17] structural model when equity and asset volatility are estimated through estimator $E ; D P\left(\Sigma_{t}^{s}\right)$ is the corresponding theoretical default probability when equity and asset volatility are $\Sigma_{t}^{s}$ and $\sigma$, respectively. Figure 4 shows the mean relative error on different calibration procedures for the noise setting (7). Similar plots are obtained for the case (6). The results for the Realized Volatility estimator using tick-by-tick data have been omitted, since the relative error in this case reaches $78 \%$ for the earliest maturities. When data are optimally sampled, the Realized Volatility calibration procedure is still affected by an error up to $6 \%$ for the shortest maturities. A negative (positive) error reveals that the calibration procedure underestimates (overestimates) default probabilities. Therefore, the classical Realized Volatility approach can severely underestimate risk. Except Fourier approach, all the other approaches slightly overestimate default probabilities, with $\Sigma_{t}^{T S}$ and $\Sigma_{t}^{K}$ providing the best estimation of risk.

Table 3 shows the corresponding numerical results suggesting that the choice of the volatility estimator largely affects the default probabilities estimation. A further extension of our study could be to analyze the magnitude of this influence when 


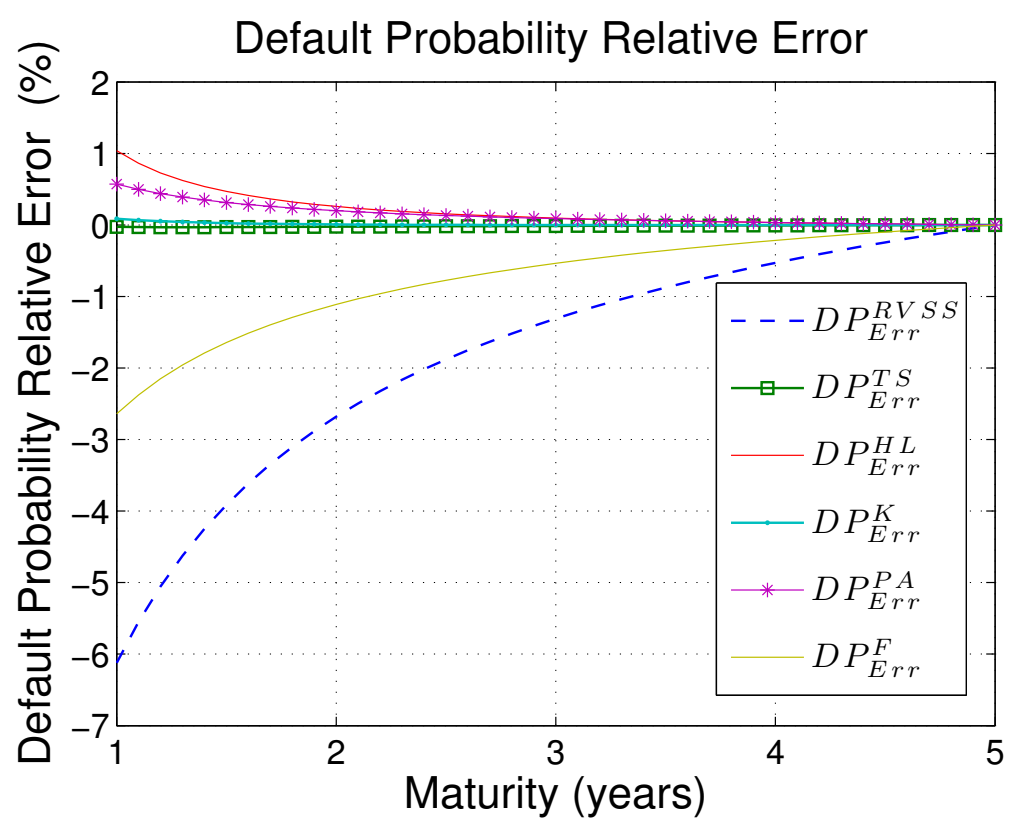

Figure 4: Default Probability Relative Error. The plot shows default probability mean relative error given in Equation (14) for maturities from 1 to 5 years. Results are based on 500 Monte Carlo simulations and refer to the introduction of a trading noise according to Equation (7), with $\alpha=5$.

considering different rating classes for corporate bonds, i.e. BB, B.

\section{Conclusions}

In this paper we consider Merton [17] structural model and use high-frequency equity prices in order to back out the unobservable asset volatility and calibrate default probabilities. We perform a Monte Carlo simulation study based on equi-spaced simulated equity prices and propose alternative equity volatility estimators, assuming data being contaminated by trading noise: the aim is to exploit the information content of intra-day high frequency data neglecting microstructure effects. While [9] propose a particle filter-based maximum likelihood method, we propose a different econometric approach. We consider alternative non-parametric (equity) volatility estimators and compare their performance in: i) filtering out the market microstructure noise, ii) extracting the (unobservable) true underlying asset volatility level, iii) predicting default probabilities. We consider, alternatively, trading noise being a) log-Gaussian distributed, b) correlated with intra-day equity log-returns. Nonobservability of a firm's asset value does not actually impede the implementation of a structural credit risk model: nevertheless, as suggested by our numerical results, the volatility estimator can largely affect calibrated default probabilities, thus risk evaluation. In particular, the commonly used Realized Volatility estimator is unable to provide reliable estimates for asset volatility under market microstructure, 
Table 3: Default Probability Relative Error. The table shows default probability mean relative error given in Equation (14) for maturities from 1 to 4.5 years. Results are based on 500 daily Monte Carlo simulations using different equity volatility estimators. Relative errors are in percentage (\%). Panel (a) refers to the trading noise given in Equation (6), panel (b) to Equation (7).

\begin{tabular}{lrrrrrrr}
\hline (a) & $D P_{E r r}^{R V}$ & $D P_{E r r}^{R V S}$ & $D P_{E r r}^{T S}$ & $D P_{E r r}^{H L}$ & $D P_{E r r}^{K}$ & $D P_{E r r}^{P A}$ & $D P_{E r r}^{F}$ \\
\hline $1 \mathrm{y}$ & -77.552877 & -5.653780 & -0.016775 & 0.678380 & 0.105648 & 0.508828 & -2.556536 \\
$1.5 \mathrm{y}$ & -59.495546 & -3.599437 & -0.022452 & 0.297156 & 0.037454 & 0.277589 & -1.590876 \\
$2 \mathrm{y}$ & -45.163526 & -2.460934 & -0.019152 & 0.160047 & 0.015950 & 0.174707 & -1.075306 \\
$2.5 \mathrm{y}$ & -33.851602 & -1.724623 & -0.014989 & 0.094462 & 0.007189 & 0.116191 & -0.748350 \\
$3 \mathrm{y}$ & -24.702511 & -1.200495 & -0.011160 & 0.057574 & 0.003154 & 0.077979 & -0.518461 \\
$3.5 \mathrm{y}$ & -17.098873 & -0.802195 & -0.007807 & 0.034536 & 0.001215 & 0.050706 & -0.345249 \\
$4 \mathrm{y}$ & -10.622653 & -0.484816 & -0.004880 & 0.019058 & 0.000322 & 0.029997 & -0.208098 \\
$4.5 \mathrm{y}$ & -4.989094 & -0.222674 & -0.002300 & 0.008090 & -0.000003 & 0.013540 & -0.095374 \\
\hline (b) & $D P_{E r r}^{R V}$ & $D P_{E r r}^{R V S S}$ & $D P_{E r r}^{T S}$ & $D P_{E r r}^{H L}$ & $D P_{E r r}^{K}$ & $D P_{E r r}^{P A}$ & $D P_{E r r}^{F}$ \\
\hline $1 \mathrm{y}$ & -78.826465 & -6.124004 & -0.027360 & 1.037514 & 0.088215 & 0.572542 & -2.640228 \\
$1.5 \mathrm{y}$ & -60.893541 & -3.913499 & -0.030161 & 0.468356 & 0.025755 & 0.314850 & -1.644234 \\
$2 \mathrm{y}$ & -46.422492 & -2.680469 & -0.024735 & 0.258533 & 0.007768 & 0.199114 & -1.111811 \\
$2.5 \mathrm{y}$ & -34.894952 & -1.880475 & -0.019030 & 0.155817 & 0.001382 & 0.132855 & -0.773942 \\
$3 \mathrm{y}$ & -25.516271 & -1.309917 & -0.014032 & 0.096727 & -0.000922 & 0.089373 & -0.536280 \\
$3.5 \mathrm{y}$ & -17.689657 & -0.875766 & -0.009755 & 0.058983 & -0.001525 & 0.058221 & -0.357158 \\
$4 \mathrm{y}$ & -11.003165 & -0.529489 & -0.006071 & 0.033040 & -0.001341 & 0.034494 & -0.215296 \\
$4.5 \mathrm{y}$ & -5.172970 & -0.243269 & -0.002852 & 0.014222 & -0.000769 & 0.015589 & -0.098680 \\
\hline
\end{tabular}

leading to a significant underestimation of asset volatility and default probabilities.

\section{References}

[1] Aït-Sahalia, Y., Mykland, P. and Zhang, L.: How often to sample a continuoustime process in the presence of market microstructure noise. Review of Financial Studies. 18, 351-416 (2005)

[2] Bandi, F.M. and Russel, J.R.: Separating market microstructure noise from volatility, Journal of Financial Economics. 79, 655-692 (2006)

[3] Bandi, F.M. and Russell, J.R.: Market microstructure noise, integrated variance estimators, and the accuracy of asymptotic approximations. Working paper, Univ. of Chicago http://faculty.chicagogsb.edu/federicobandi (2006)

[4] Barndorff-Nielsen, O.E., Hansen, P.R., Lunde, A. and Shephard, N.: Designing realised kernels to measure the ex-post variation of equity prices in the presence of noise. Econometrica. 76:6, 1481-1536 (2008)

[5] Barndorff-Nielsen, O.E., Hansen, P.R., Lunde, A. and Shephard, N.: Multivariate realised kernels: consistent positive semi-definite estimators of the covariation of equity prices with noise and non-synchronous trading. Working paper (2008) 
[6] Barndorff-Nielsen, O.E., Hansen, P.R., Lunde, A. and Shephard, N.: Subsampling realised kernels. Journal of Econometrics, in press (2010)

[7] Barndorff-Nielsen, O.E. and Shephard, N.: Econometric analysis of realized volatility and its use in estimating stochastic volatility models. J. R. Statist. Soc., Ser. B, 64, 253-280 (2002)

[8] Duan J.C.: Maximum likelihood estimation using price data of the derivative contract. Mathematical Finance. 4, 155-167 (1994)

[9] Duan J.C., Fulop A.: Estimating the structural credit risk model when equity prices are contaminated by trading noises. Journal of Econometrics. 150, 288296 (2009)

[10] Ericsson J., Reneby J.: Estimating Structural Bond Pricing Models. Journal of Business. 78, 2, 707-735 (2005)

[11] Hansen, P.R. and Lunde, A.: Realized variance and market microstructure noise (with discussions). Journal of Business and Economic Statistics. 24, 127218 (2006)

[12] Huang J., Huang M.: How much of the corporate-treasury yield spread is due to credit risk?. Working Paper. Penn State University (2003)

[13] Jacod, J., Li. Y., Mykland, P.A., Podolskij, M. and Vetter, M.: Microstructure noise in the continuous case: the pre-averaging approach. Stochastic Processes and their Applications. 119, 2249-2276 (2009)

[14] Jones, Philip E., Scott P. Mason, and Eric Rosenfeld: Contingent claims analysis of corporate capital structures: an empirical investigation. Journal of Finance. 39, 611-625 (1984)

[15] Malliavin P., Mancino M.E.: Fourier series method for measurement of multivariate volatilities. Finance and Stochastics. Springer, 6(1), pages 49-61 (2009)

[16] Mancino M.E., Sanfelici S.: Robustness of Fourier estimator of integrated volatility in the presence of microstructure noise. Computational Statistics \& Data analysis. Elsevier, 52, 2966-2989 (2008)

[17] Merton R. C.: On the Pricing of Corporate Debt: The Risk Structure of Interest Rates, The Journal of Finance. 29, 449-470 (1974)

[18] Eom Y.H., Helwege J., Huang J.: Structural models of corporate bond pricing: an empirical analysis. Review of Financial Studies. 17, 499-544 (2008)

[19] Zhang, L., Mykland, P. and Ait-Sahalia, Y.: A tale of two time scales: determining integrated volatility with noisy high frequency data. Journal of the American Statistical Association. 100, 1394-1411 (2005)

[20] Zhang B.Y., Zhou H., Zhu H.: Explaining Credit Default Swap Spreads with the Equity Volatility and Jump Risks of Individual Firms. Review of Financial Studies. 22:12, 5099-5131 (2009) 